\title{
STARS DO NOT EAT THEIR YOUNG MIGRATING PLANETS: EMPIRICAL CONSTRAINTS ON PLANET MIGRATION HALTING MECHANISMS
}

\author{
Peter Plavchan ${ }^{1}$ and Christopher Bilinski ${ }^{2}$ \\ ${ }^{1}$ NASA Exoplanet Science Institute, California Institute of Technology, M/C 100-22, 770 South Wilson Avenue, Pasadena, CA 91125, USA \\ ${ }^{2}$ University of Arizona, Tucson, AZ 85721, USA \\ Received 2011 December 7; accepted 2013 March 23; published 2013 May 7
}

\begin{abstract}
The discovery of "hot Jupiters" very close to their parent stars confirmed that Jovian planets migrate inward via several potential mechanisms. We present empirical constraints on planet migration halting mechanisms. We compute model density functions of close-in exoplanets in the orbital semi-major axis-stellar mass plane to represent planet migration that is halted via several mechanisms, including the interior 1:2 resonance with the magnetospheric disk truncation radius, the interior 1:2 resonance with the dust sublimation radius, and several scenarios for tidal halting. The models differ in the predicted power-law dependence of the exoplanet orbital semi-major axis as a function of stellar mass, and thus we also include a power-law model with the exponent as a free parameter. We use a Bayesian analysis to assess the model success in reproducing empirical distributions of confirmed exoplanets and Kepler candidates that orbit interior to $0.1 \mathrm{AU}$. Our results confirm a correlation of the halting distance with stellar mass. Tidal halting provides the best fit to the empirical distribution of confirmed Jovian exoplanets at a statistically robust level, consistent with the Kozai mechanism and the spin-orbit misalignment of a substantial fraction of hot Jupiters. We can rule out migration halting at the interior 1:2 resonances with the magnetospheric disk truncation radius and the interior 1:2 resonance with the dust disk sublimation radius, a uniform random distribution, and a distribution with no dependence on stellar mass. Note that our results do not rule out Type-II migration, but rather eliminate the role of a circumstellar disk in stopping exoplanet migration. For Kepler candidates, which have a more restricted range in stellar mass compared to confirmed planets, we are unable to discern between the tidal dissipation and magnetospheric disk truncation braking mechanisms at a statistically significant level. The power-law model favors exponents in the range of 0.38-0.9. This is larger than that predicted for tidal halting (0.23-0.33), which suggests that additional physics may be missing in the tidal halting theory.
\end{abstract}

Key words: planet-disk interactions - planet-star interactions - protoplanetary disks

Online-only material: color figure

\section{INTRODUCTION}

Many "hot Jupiter" planets have been discovered to orbit very close to their central stars (e.g., Marcy \& Butler 1998; Marcy et al. 1997; Borucki et al. 2011). It is well established that these planets must form further out from their host stars, likely beyond the snow line, and either migrate embedded in a primordial disk or via dynamical interactions (e.g., Lin et al. 1996; Lubow \& Ida 2010; Kozai 1962; Triaud et al. 2010). Observations of the Rossiter-McLaughlin effect to identify stellar spin-planet orbit misalignment show that a significant fraction of "hot Jupiters" are aligned, and a significant fraction are also misaligned (Morton \& Johnson 2011; Triaud et al. 2010; Narita et al. 2010). The misaligned planets are likely directed inward via planet-planet scattering, the Kozai mechanism, secular chaos, or analogous mechanisms (e.g., Kozai 1962; Wu \& Murray 2003; Naoz et al. 2011; Nagasawa \& Ida 2011; Wu \& Lithwick 2011). Recent work by Dawson et al. (2013) and Dawson \& Murray-Clay (2013) suggests that less than 15\% of hot Jupiters undergo migration via the Kozai mechanism, instead favoring planet-planet scattering.

For aligned close-in planets, planet migration embedded in a primordial disk is suspected to explain the observed planet location. Most disk migration models involve similar physical processes, but contain differences in the underlying assumptions about the structure and properties of the primordial disk and the planet (e.g., viscosity, density, scale height, temperature, dissipation timescale, toroidal magnetic fields embedded in the disk, etc.; Lin et al. 1996; Lubow \& Ida 2010; Raymond et al. 2006; Menou \& Goodman 2004; Tanaka et al. 2002; Terquem 2003). Current hypotheses often combine a few models together in an attempt to explain observed exoplanet mass and semimajor axis distributions. The justification cited is that conditions within the disk and the planet mass and density change over time, resulting in different models being applicable at different times in the planet migration process.

Type-I migration assumes that the density structure of the disk is not affected by planets. Instead, turbulence determines the density structure of the disk. For this reason, this form of migration is most applicable to small mass planets. In the case of Type-II migration, a gap is formed between the disk and a high-mass planet. This gap is the result of the tidal torques from the planet becoming stronger than the viscous torques of the disk. Initial theoretical models of Type-I and Type-II migration suggested a rapid planet migration rate that could result in planet destruction by dispersing the accreted material (Ward 1997a, 1997b). Various mechanisms are thought to decrease the planet migration rate, including eccentric planet orbits and disk turbulence (Lubow \& Ida 2010; Menou \& Goodman 2004). In all cases, planet-disk migration must take place while the primordial disk of gas and dust is still present during the classical T Tauri phase, or first $\sim 5 \mathrm{Myr}$, of the host star's life (Silverstone et al. 2006; Currie et al. 2009).

Type-II migration offers a mechanism to transport gas giants that must form beyond the snow line inward to their host star. However, it is not well constrained how planet migration 
is halted once started, lest the planet be tidally disrupted by the host star. Possible braking mechanisms include tidal circularization (Ford \& Rasio 2006; Wu et al. 2007; Guillochon et al. 2011; Arras et al. 2012; Matsumura et al. 2010; Lai 2012), trapping the exoplanet in the 1:2 interior orbital resonance with the magnetospheric truncation radius (Eisner et al. 2005), or trapping the planet in the 1:2 interior orbital resonance with the dust sublimation radius (e.g., Kuchner \& Lecar 2002). For an approximately solar-mass star, the gas disk truncation radius is comparable to the dust sublimation radius for a typical $\mathrm{T}$ Tauri star magnetic field strength of $\sim 2 \mathrm{kG}$ (Eisner et al. 2005), but that is not the case for lower- and higher-mass stars. For lower(higher-)mass T Tauri stars, the dust sublimation radius can be interior (exterior) to the estimated magnetospheric truncation radius (Section 3).

In this work, we investigate exoplanet distributions as a function of semi-major axis and host stellar mass as a test for migration halting mechanisms. The increasing number of exoplanet discoveries provides sufficiently large ensembles of close-in (e.g., $<0.1 \mathrm{AU}$ ) exoplanets over a range of host stellar masses to discern which mechanism may be responsible for halting exoplanet migration. In Section 2, we outline our empirical samples. In Section 3, we present each migration halting mechanism model and its corresponding prediction for the exoplanet distribution density function. In Section 4, we present our methodology to evaluate the success of each migration halting model at reproducing empirical distributions, and in Section 5, we present the results of these statistical tests. In Section 6, we present our conclusions.

\section{EMPIRICAL SAMPLES}

We make use of two empirical samples_confirmed exoplanets as of 2012 February with $M_{\mathrm{pl}}<30 M_{J}$ (Akeson et al. 2013; Wright et al. 2011), and the third tabulation of Kepler planetary candidates, also known as Kepler Objects of Interest (KOI; Batalha et al. 2013; Borucki et al. 2011; Howard et al. 2012). We further sub-divide the confirmed planets by mass $\mathrm{M}_{\mathrm{pl}}$ (or $m \sin i$ ) into mass bins of $M_{\mathrm{pl}}<10 M_{\oplus}, 10 M_{\oplus}<M_{\mathrm{pl}}<0.2 M_{J}$, and $M_{\mathrm{pl}}>0.2 M_{J}$. We also further sub-divide the Kepler candidates by estimated planet radius into three radius bins with $R_{\mathrm{pl}}<2 R_{\oplus}, 2 R_{\oplus}<R_{\mathrm{pl}}<6 R_{\oplus}$, and $R_{\mathrm{pl}}>6 R_{\oplus}$. These subdivisions are chosen to approximate the terrestrial, super Earth/ Neptune, and Jovian planet mass/radius boundaries. Estimates of stellar mass are culled from the literature from the NASA Exoplanet Archive and exoplanets.org for the confirmed exoplanets. The revised stellar masses from Batalha et al. (2013) are utilized for the Kepler candidates, rather than the Kepler Input Catalog (Brown et al. 2011). These six empirical samples are shown in Figure 1.

We exclude all exoplanets and candidates with orbital semimajor axes $>0.1$ AU to focus on close-in planets most likely to have undergone some form of migration in their orbital evolution, rather than forming in situ at their present locations. We also constrain our samples to stellar masses between 0.1 and $1.5 M_{\odot}$. The upper limit of $1.5 M_{\odot}$ is chosen to exclude planets around higher-mass stars that can be evolved sub-giants (e.g., Johnson et al. 2011). The final exoplanet counts in each of our samples are: 203 confirmed exoplanets and 1199 Kepler candidates, including $115 \mathrm{KOIs}$ with $R_{\mathrm{pl}}>6 R_{\oplus}, 434$ with $2 R_{\oplus}<R_{\mathrm{pl}}<6 R_{\oplus}$, and 650 with $R_{\mathrm{pl}}<2 R_{\oplus}$.

Inherent in these samples are many survey biases and incompleteness. We discuss these briefly. The frequency of planets as a function of stellar mass is highly dependent on the survey sample selection criteria of ongoing searches. For example, the Kepler Input Catalog was selected to focus on FGK-type stars, with a paucity of M dwarfs (Batalha et al. 2010a, 2010b; Borucki et al. 2011), and visible radial velocity searches initially focused on similar solar-mass stars but now include smaller samples of lower- and higher-mass stars (Marcy \& Butler 1998). Additionally, there are differences in the planet frequency as a function of planet mass and stellar mass that are not yet well constrained (e.g., Howard et al. 2012). Thus, in our analysis that follows, we fix our models to match the empirical frequency of exoplanets as a function of stellar mass (within 0.1 AU for a given data set). Further, we do not draw any conclusions about the planet frequency as a function of stellar mass.

The transit and radial velocity detection methods, responsible for the discovery of most close-in confirmed exoplanets, are highly biased toward the detection of short-period orbits. Additionally, at a fixed semi-major axis, planets around a lower-mass star will have a longer orbital period. This introduces bias toward a higher detection efficiency toward higher stellar masses at a fixed semi-major axis. However, most ground-based surveys are reasonably complete to within our semi-major axis cut of $0.1 \mathrm{AU}$, corresponding to an orbital period of $\sim 16$ days for a solar-mass star. After more than two years of operation, Kepler is also complete out to $0.1 \mathrm{AU}$, down to some nominal terrestrial planet size (Howard et al. 2012; Batalha et al. 2013). Thus, we do not expect detection completeness as a function of semi-major axis to significantly impact our analysis.

For the confirmed exoplanet sample, we do not apply any minimum constraint on planet mass (or planet-mass limit in the case of radial velocity detected exoplanets). While terrestrial planets are more likely to have formed in situ rather than migrated inward to their present locations, most radial velocity confirmed exoplanets are Neptune-massed or larger, with a few super Earths. The presence of a few lowmass planets in our sample does not significantly impact our analysis.

Finally, we also do not correct for the false-positive rate in the Kepler exoplanet candidate list, which is thought to be between $10 \%$ and $35 \%$, but may be particularly high for orbital periods of $<3$ days, where background eclipsing binaries are more likely to mimic the signature of a hot Jupiter (Howard et al. 2012; Borucki et al. 2011; Morton \& Johnson 2011; Plavchan et al. 2013; Santerne et al. 2012). Future releases of the Kepler candidates including follow-up identification of false positives and improved completeness at short orbital periods will improve our analysis presented herein, but accounting for the false positives in the current KOI list is beyond the scope of this work.

\section{MIGRATION HALTING MODELS}

For each migration halting mechanism model we present in this section, our goal is to generate a reasonably simple prediction for the density of exoplanets as a function of stellar mass and semi-major axis within 0.1 AU. We will present the model for each mechanism in turn, but first we outline commonalities in our methodology across models.

With the exception of the uniform random and exponential decay distribution models, our prescription involves first identifying a single 1:1 curve in the semi-major axis-stellar mass plane. The specification of these curves is outlined in Sections 3.1-3.4 for each particular model. We next convolve that curve with a Gaussian kernel in the log of the orbital semi-major axis to 


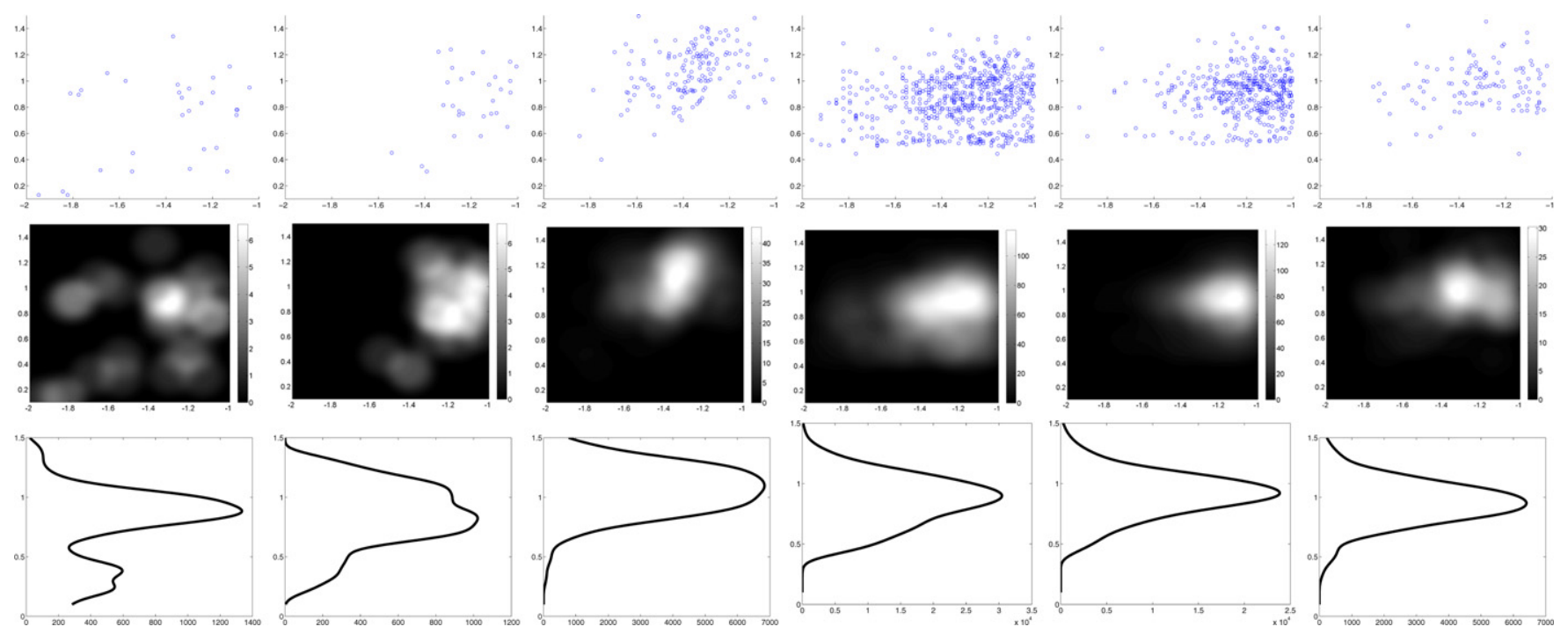

Figure 1. First row: empirical distributions of exoplanet semi-major axes and host star masses for (from left to right): confirmed exoplanets as of 2012 February (NASA Exoplanet Archive) with $M_{\mathrm{pl}}<10 M_{\oplus}$, confirmed exoplanets with $10 M_{\oplus}<M_{\mathrm{pl}}<0.3 M_{J}$, confirmed exoplanets with $M_{\mathrm{pl}}>0.3 M_{J}$, Kepler Objects of Interest from Batalha et al. (2013) with $R_{\mathrm{pl}}<2 R_{\oplus}$, Kepler Objects of Interest with $2 R_{\oplus} \leqslant R_{\mathrm{pl}} \leqslant 6 R_{\oplus}$, and Kepler Objects of Interest with $R_{\mathrm{pl}}>6 R_{\oplus}$. Second row: empirical density functions generated for each data set using a method analogous to that in Wasserman (2005). Third row: planet frequency as a function of stellar mass for each data set in the first row, shown with arbitrary normalization. This is used as a normalization for our migration braking models to remove survey sample selection effects as a function of stellar mass.

(A color version of this figure is available in the online journal.)

arrive at a predicted probability density function (PDF) to compare with an empirical distribution. The Gaussian width is a free parameter optimized for each model and empirical distribution combination we test. The use of a Gaussian kernel is an ad hoc step in our model generation, but its use is motivated by its simplicity and the observed scatter of exoplanet orbital semimajor axes.

Each model is next divided by the empirical frequency of exoplanets (within $0.1 \mathrm{AU}$ ) as a function of stellar mass to correct for sample survey biases which are not relevant to our investigation herein (Figure 1). Finally, for computational simplicity, the PDF for each model is evaluated numerically on a $500 \times 500$ grid evenly spaced in stellar mass from 0.1 to $1.5 M_{\odot}$ and in the log of the orbital semi-major axis in $\mathrm{AU}$ from $\log (a)=-2$ to -1 . The model density functions are summed over all pixels and the sum is normalized to 1 .

\subsection{Tidal Dissipation}

Planet-planet scattering, secular chaos, and the Kozai cycle in particular are theoretical mechanisms proposed to migrate a Jovian exoplanet inward toward its host star (Kozai 1962; Naoz et al. 2011; Nagasawa \& Ida 2011; Wu \& Lithwick 2011; Wu \& Murray 2003). These mechanisms invoke tidal forces acting on the exoplanet to lower the semi-major axis and eccentricity until the orbit is circularized. Some of these mechanisms are likely required to explain the known fraction of spin-orbit misaligned closein exoplanets (Morton \& Johnson 2011; Triaud et al. 2010; Albrecht et al. 2011, 2012; Winn et al. 2010). The association of the observed spin-orbit misalignment with the Kozai and similar migration mechanisms relies on the assumption that the stellar spin axis is aligned with the primordial disk rotation axis. This star-disk alignment is intuitively expected from the process of star formation (Prato \& Weinberger 2007; Hale 1994; Watson et al. 2011 and references therein), although mechanisms such as an external perturber are proposed to alter this alignment (Kaib et al. 2011; Thies et al. 2011). In order for tidal circularization to occur, the exoplanet must get within several stellar radii of the parent star, i.e., within a few tenths of an AU (Arras et al. 2012). Tidal circularization predicts closerin orbits around lower-mass stars with deeper convective atmospheres (Kozai 1962; Wu \& Murray 2003; Arras et al. 2012).

We include two theoretical predictions for the stopping radius as a function of stellar mass from tidal circularization. First, Ford \& Rasio (2006) predict a minimum allowable envelope for the exoplanet semi-major axis scaling from the Roche radius, with a scaling with stellar mass of $a \propto M_{*}^{1 / 3}$. Guillochon et al. (2011) suggest that the particular proportionality constant should be increased over that in Ford \& Rasio (2006). Second, Wu et al. (2007, Equation (6)) predict the final semi-major axis itself, scaling with a slightly different dependence on stellar mass of $a \propto M_{*}^{3 / 13}$ and weakly dependent on the unknown planet resonant $Q$ factor. In both of our models used in our analysis, we allow for the proportionality constant $C$ as a free parameter, only fixing the power-law exponent $(\alpha=1 / 3$ or $3 / 13$ ). The proportionality constant and Gaussian kernel used to generate the exoplanet density function in these models can be interpreted to represent a degenerate range of exoplanet and/or stellar densities $/ Q$ 's and initial conditions about these assumed mean values.

\subsection{The Interior 1:2 Orbital Resonance with the Accretion Disk Truncation at the Magnetospheric Radius}

The magnetosphere of a T Tauri star has long been thought to truncate the inner accreting primordial gas disk (e.g., Chiang \& Goldreich 1997; Meyer et al. 1997), and recent interferometric observations of young stars confirm these inner holes exist (Eisner et al. 2005). A Jovian exoplanet undergoing Type-II migration in a primordial disk could halt after it enters into this inner clearing, as has been proposed (Eisner et al. 2005; Kuchner \& Lecar 2002; Lin et al. 1996). In this scenario, a migrating Jovian exoplanet interacts with a protoplanetary disk at Lindblad resonances, transferring angular momentum 
via torques to the disk as the planet migrates inward. When the 2:1 Lindblad resonance site enters the evacuated portion of the disk, the planet can no longer transfer angular momentum to the disk and the migration is hypothesized to halt. The exoplanet continues to orbit the host star at a period equal to onehalf of the Keplerian orbital period of the magnetospheric gas disk truncation radius. A related but distinct halting mechanism is proposed for smaller planets undergoing Type-I migration in Tsang (2011).

The magnetospheric radius can be approximated by (Eisner et al. 2005; Konigl 1991)

$$
R_{\mathrm{mag}}=2.27 R_{1}\left[\frac{\left(B_{0} / 1 \mathrm{kG}\right)^{4}\left(R_{*} / R_{\odot}\right)^{5}}{\left(M_{*} / M_{\odot}\right)\left(\dot{M} /\left(10^{-7} M_{\odot} \mathrm{yr}^{-1}\right)\right)^{2}}\right]^{1 / 7}
$$

where $B_{0}$ is the stellar magnetic field strength. A field strength of $2 \mathrm{kG}$ is typical for T Tauri stars (Johns-Krull et al. 2003), and is a free parameter in our model density function. $\dot{M}$ is the stellar accretion rate in units of $M_{\odot} \mathrm{yr}^{-1}$, which can vary by several orders of magnitude for young stars. Muzerolle et al. (2003) derive an approximate power-law relationship between stellar mass and an accretion rate of $\dot{M} \propto M^{2}$. We estimate a proportionality constant of $10^{-8.5} M_{\odot} \mathrm{yr}^{-1}$ that yields appropriate accretion rates for solar-type stars as inferred from Muzerolle et al. (2003, Figure 8). For a $2 \mathrm{kG}$ field, the magnetospheric truncation radius is thus approximately given by

$$
R_{\mathrm{mag}}=9.05 R_{\odot}\left(\frac{R_{*}}{R_{\odot}}\right)^{12 / 7}\left(\frac{M_{\odot}}{M_{*}}\right)^{5 / 7} \approx 9 R_{*} .
$$

The 1:2 interior orbital resonance with this inferred magnetospheric radius is thus located at $\sim 6 R_{*}$ for a $2 \mathrm{kG}$ stellar magnetic field, and our full migration halting semi-major axis location, $a$, is given by

$$
\begin{aligned}
a= & 9.05 R_{\odot}\left(\frac{B_{0}}{2 \mathrm{kG}}\right)^{4 / 7}\left(\frac{R_{*}}{R_{\odot}}\right)^{12 / 7} \\
& \times *\left(\frac{M_{\odot}}{M_{*}}\right)^{5 / 7} * \frac{1}{2} \propto M^{1 / 7} .
\end{aligned}
$$

Finally, to arrive at our model analytic curve, we assume $\log g=4$ as is typical for T Tauri stars that are still contracting onto the main sequence (Greene \& Lada 1996). With these assumptions, we can express this migration halting radius as a function of only $B_{0}$ and $M_{*}$. The Gaussian kernel that we use to generate the model exoplanet density function from the analytic curve in Equation (3) can be interpreted to represent the degenerate range in magnetic field strengths, stellar accretion rates, and/or stellar surface gravity about the assumed values. For example, the range of observed $\mathrm{T}$ Tauri accretion rates ( 100; Muzerolle et al. 2003) would correspond to a range for $a$ in Equation (1) of a factor of $\sim 2$. Additionally, the pre-main-sequence contraction times imply that the median magnetic field strength should vary as a function of stellar mass due to the range of different evolutionary states at a fixed protostellar age. Finally, exoplanet Type-II migration may also preferentially take place at different stellar ages as a function of stellar mass (Lubow \& Ida 2010; Lin et al. 1996). In our analysis that follows, however, we assume that we are only varying the magnetic field strength, and we keep the median magnetic field strength constant as a function of stellar mass. At this time, we do not overcomplicate our model to account for these various degenerate factors (Section 5).

\subsection{The Interior 1:2 Orbital Resonance with the Dust Disk Sublimation Radius}

The truncation of the dust disk at the dust sublimation radius is also proposed as a mechanism to halt the inward migration of Jovian planets for solar-type stars (Kuchner \& Lecar 2002; Lin et al. 1996). This scenario for migration halting is identical to that for the magnetospheric truncation model in Section 3.1, except the planet is hypothesized to halt instead at the interior 1:2 orbital resonances with the dust disk sublimation radius. While the primordial (gas and dust) disk for a typical $\mathrm{T}$ Tauri star is thought to dissipate by a stellar age of $\sim 5 \mathrm{Myr}$, a debris dust disk from the collision of planetesimals can persist for much longer. For a solar-mass star, the dust sublimation radius is approximately equal to the expected magnetospheric gas disk truncation radius, and thus solar-type stars alone are a poor discriminator of migration halting mechanisms. However, the dust sublimation radius has a significantly different dependence on stellar mass when compared to the magnetospheric gas disk truncation radius.

The dust sublimation radius is approximately given by the expression (Jura et al. 1998)

$$
R_{\text {subl }}=\frac{1}{2} R_{*}\left(\frac{T_{*}}{1500}\right)^{2}
$$

where $T_{*}$ is the effective temperature of the host star in Kelvin, $R_{*}$ is the radius of the host star, and we have assumed a dust sublimation temperature of $1500 \mathrm{~K}$. Equation (4) assumes that the dust can be approximated by a blackbody in local thermal equilibrium, and that the dust is optically thin to the incident stellar radiation. Assuming to the contrary for both factors would decrease the dust sublimation radius. Viscous heating in a primordial disk can increase the dust sublimation radius, and more sophisticated treatments yield a stronger dependence of the sublimation radius on the stellar temperature (Robitaille et al. 2006, 2007; D'Alessio et al. 2006). In our analysis, we retain the approximation in Equation (4) for simplicity, but also because of the lack of success for this particular model (Section 5).

We adopt a temperature-radius relation using a Siess et al. (2000) 10 Myr isochrone to express the migration halting radius, $a$, as a function of only the stellar radius, e.g.,

$$
a=\frac{1}{2}^{5 / 3}\left(\frac{T_{* \operatorname{Siess}}\left(R_{*}\right)}{1500}\right)^{2} R_{*},
$$

where the extra factor of $1 / 2^{2 / 3}$ comes from Kepler's laws and the 1:2 interior resonance location with respect to the dust sublimation radius in Equation (5). Our results are not significantly altered if we instead use a $10 \mathrm{Myr}$ isochrone from Baraffe et al. (1998).

We adopt the age isochrone of $10 \mathrm{Myr}$ with the assumption that the exoplanet migration under this scenario must take place early in a star's evolution. An older stellar age will decrease the modeled migration halting radius in Equation (5), since the star will continue to contract onto the main sequence. Finally, to arrive at our model analytic curve, we again assume $\log g=4$ in order to express this migration halting radius as a function of only $M_{*}$. The Gaussian kernel that we use to generate the model exoplanet density function from Equation (5) can be interpreted to represent a degenerate range in stellar surface gravity and/or dust sublimation temperatures about the assumed values.

Since the stellar effective temperature varies little over the main-sequence lifetime of the host star, we can also estimate 
the distance $a$ in Equation (5) for older main-sequence stars. For main-sequence $\mathrm{M}$ dwarfs, we note that the semi-major axis in Equation (5) for this orbital resonance is less than $\sim 2.2 R_{*}$. This orbital separation falls within the estimated Roche radius of $\sim 2.4 R_{*}$ (Section 3.1), and a planet at this distance would likely be tidally disrupted. We conclude that no migrating Jovian planets would survive around $\mathrm{M}$ dwarfs if the orbital resonance with the dust sublimation radius is responsible for braking Jovian planet migration, and if the migration takes place after the $\mathrm{M}$ dwarfs have reached the main sequence. The expected exoplanet detection frequency for this scenario is zero for $\mathrm{M}$ dwarfs. While this is an interesting potential mechanism to explain the observed lack of $\mathrm{M}$ dwarf hot Jupiters relative to solar-mass stars (Endl et al. 2006; Plavchan et al. 2008), we do not find this scenario likely given our results in Section 5.

\subsection{Power Law and Other Models}

The models described in the previous sections predict a distribution of exoplanets in the stellar mass-semi-major axis plane that fits a particular choice for the exponent $\alpha$ in a powerlaw model. To ensure we are considering a more complete range of models, we also carry out a separate analysis with a powerlaw model where the exponent $\alpha$ is a free parameter. Again, we also include a Gaussian kernel width $\sigma$ and a proportionality constant $C$ as free parameters in this model.

Next, we include three additional models as a sanity check on our analysis. The first assumes that the exoplanets within 0.1 AU are uniform randomly distributed as a function of stellar mass and semi-major axis. The second assumes a uniform random dependence on stellar mass and an exponential decaying dependence on semi-major axis-e.g., favoring smaller semimajor axes in a fashion that is uniform random in $\log (a)$. The third model assumes that exoplanets halt at a constant semimajor axis $A$ that is independent of the stellar mass of the star-e.g., a power-law model with an exponent of zero. A Gaussian kernel width is included as a free parameter to generate the final PDF as with previous models.

\section{METHODOLOGY FOR EVALUATING MODEL SUCCESS}

We use a variety of different free parameters for our models as described in Section 3. To evaluate which migration braking mechanism is best at reproducing the different empirical distributions and to determine optimal parameters, we use two approaches. We discuss each in turn.

\subsection{Bayesian Evaluation}

We use a Bayesian analysis to estimate the posterior probability $P\left(H_{i} \mid D\right)$ of obtaining a given empirical data set $D=\left\{D_{j}\right\}$ for a particular migration braking model hypothesis $H_{i}$. Our approach is similar to that in Marshall et al. (2006). Explicitly, we re-state Bayes' theorem:

$$
P\left(H_{i} \mid D\right)=\frac{P\left(H_{i}\right) P\left(D \mid H_{i}\right)}{P(D)} .
$$

To optimize the parameter selection for a given empirical data set $D$ and model $H_{i}$, we maximize the posterior probability $P\left(H_{i} \mid D\right)$ for the same model over a range of its free parameters.

Following the Bayesian technique, we obtain the likelihoods $P\left(D \mid H_{i}\right)$ by multiplying together the individual PDFs
$\operatorname{PDF}_{H_{i}}\left(D_{j}\right)$ to calculate the probability of obtaining a single $D_{j}$ :

$$
P\left(D \mid H_{i}\right)=\prod_{j} \operatorname{PDF}_{H_{i}}\left(D_{j}\right)
$$

where $D_{j}$ is the semi-major axis and stellar host mass value pair for an individual exoplanet. We use the probability densities as described in Section 3 to estimate each individual $\operatorname{PDF}_{H_{i}}\left(D_{j}\right)$, linearly interpolating from the $500 \times 500$ model grid.

For the prior terms, $P\left(H_{i}\right)$, we make some assumptions. First, we use a uninformed Jeffrey's prior of $1 / \sigma$ (e.g., $P\left(H_{i}\right) \propto$ $\left.\left(1 / \sigma_{i}\right)\right)$ for the Gaussian width $\sigma$ parameter present in a number of the models. To normalize a given model prior such that $\sum_{i} P\left(H_{i}\right)=1$ in the discrete limit, we determined that $P\left(H_{i}\right)=$ $\left(C / \sigma_{i}\right)$, where $C=1 / \sum_{i}\left(1 / \sigma_{i}\right)$, and $\left\{\sigma_{i}\right\}$ are the discrete set of widths evaluated in our analysis from 0.02 to 1.02 in steps of 0.02. Next, we also assume uninformed Jeffrey's priors for the $\log$ of $B_{0}, C$, and $A$ parameters in the magnetospheric disk truncation, power law, and stellar-mass-independent models, respectively, discretely evaluated with 51 steps between $a=$ 0.01 and 0.11 . Finally, we assume a uniform random prior for $\alpha$ in the power-law model, discretely evaluated between 0 and 1 with steps of 0.02 . The bounds for our parameter space exploration are not preferred as optimal values for our models, with the exception of the value of $\sigma$ for the dust sublimation halting model which is particularly inadequate in describing the empirical data sets.

The last term in Bayes' theorem needed to compute the posterior probabilities (evidences) is the marginal probability, $P(D)$. However, we can rewrite this term as

$$
P(D)=\sum_{i} P\left(D \mid H_{i}\right) P\left(H_{i}\right),
$$

where we sum over all hypotheses, $i$. We do not have an exhaustive (complete) list of models. Additionally, we include an arbitrary normalization $N$ in our analysis to avoid the double data type machine precision limit. Thus, we do not obtain absolute posterior probabilities, and we can end up with relative posterior probabilities greater than one. Nevertheless, we know that the value of $N / P(D)$ is the same in all of our computations, so we can ignore it and compute accurate relative posterior probabilities when we are comparing parameters for a given model or between models. In other words, the quantity of interest is

$$
\begin{aligned}
\frac{P\left(H_{1} \mid D\right)}{P\left(H_{2} \mid D\right)} & =\frac{N P\left(H_{1}\right) P\left(D \mid H_{1}\right)}{P(D)} \times \frac{P(D)}{N P\left(H_{2}\right) P\left(D \mid H_{2}\right)} \\
& =\frac{P\left(H_{1}\right) P\left(D \mid H_{1}\right)}{P\left(H_{2}\right) P\left(D \mid H_{2}\right)} .
\end{aligned}
$$

This approach also enables a relative comparison of models that factors in the degrees of freedom and the ranges of explored parameter space for a given model.

\subsection{Chi-squared Tests}

In order to test the predicted exoplanet density function against the empirical planet distributions using the chi-squared test, we first generate a density function for the empirical planet distributions. This was not necessary for the Bayesian analysis, which directly tested the empirical distribution of exoplanets against the model density function.

The density function is calculated for each data set using a kernel density estimation method analogous to that in Wasserman 
Table 1

Model Best-fit Parameter Values ${ }^{\mathrm{a}}$

\begin{tabular}{|c|c|c|c|c|c|c|}
\hline Model & $M_{\mathrm{pl}}<10 M_{\oplus}$ & $\begin{array}{c}10 M_{\oplus}<M_{\mathrm{pl}}< \\
0.3 M_{J}\end{array}$ & $M_{\mathrm{pl}}>0.3 M_{J}$ & $R_{\mathrm{pl}}<2 R_{\oplus}$ & $\begin{array}{c}2 R_{\oplus} \leqslant R_{\mathrm{pl}} \leqslant \\
6 R_{\oplus}\end{array}$ & $R_{\mathrm{pl}}>6 R_{\oplus}$ \\
\hline$M^{1 / 3}$ tidal model & $\begin{array}{c}C=0.052 \\
\sigma=0.34\end{array}$ & $\begin{array}{c}C=0.072 \\
\sigma=0.14\end{array}$ & $\begin{array}{c}C=0.042 \\
\sigma=0.16\end{array}$ & $\begin{array}{c}C=0.07 \\
\sigma=0.3\end{array}$ & $\begin{array}{c}C=0.102 \\
\sigma=0.26\end{array}$ & $\begin{array}{c}C=0.056 \\
\sigma=0.24\end{array}$ \\
\hline$M^{3 / 13}$ tidal model & $\begin{array}{l}C=0.05 \\
\sigma=0.36\end{array}$ & $\begin{array}{l}C=0.07 \\
\sigma=0.14\end{array}$ & $\begin{array}{c}C=0.044 \\
\sigma=0.16\end{array}$ & $\begin{array}{c}C=0.068 \\
\sigma=0.3\end{array}$ & $\begin{array}{l}C=0.1 \\
\sigma=0.26\end{array}$ & $\begin{array}{c}C=0.056 \\
\sigma=0.24\end{array}$ \\
\hline Magnetospheric truncation & $\begin{array}{c}B_{0}=1.1547 \mathrm{kG} \\
\sigma=0.38\end{array}$ & $\begin{array}{c}B_{0}=2.4075 \mathrm{kG} \\
\sigma=0.16\end{array}$ & $\begin{array}{c}B_{0}=1.0683 \mathrm{kG} \\
\sigma=0.16\end{array}$ & $\begin{array}{c}B_{0}=2.2884 \mathrm{kG} \\
\sigma=0.3\end{array}$ & $\begin{array}{c}B_{0}=4.338 \mathrm{kG} \\
\sigma=0.26\end{array}$ & $\begin{array}{c}B_{0}=1.6292 \mathrm{kG} \\
\sigma=0.24\end{array}$ \\
\hline Dust sublimation & $\sigma=1.02$ & $\sigma=1.02$ & $\sigma=0.86$ & $\sigma=1.02$ & $\sigma=1.02$ & $\sigma=1.02$ \\
\hline Power law & $\begin{array}{c}\alpha=0.9 \\
C=0.062 \\
\sigma=0.34\end{array}$ & $\begin{array}{c}\alpha=0.58 \\
C=0.078 \\
\sigma=0.14\end{array}$ & $\begin{array}{c}\alpha=0.68 \\
C=0.042 \\
\sigma=0.16\end{array}$ & $\begin{array}{c}\alpha=0.6 \\
C=0.074 \\
\sigma=0.3\end{array}$ & $\begin{array}{c}\alpha=0.38 \\
C=0.104 \\
\sigma=0.26\end{array}$ & $\begin{array}{c}\alpha=0.4 \\
C=0.058 \\
\sigma=0.24\end{array}$ \\
\hline Uniform random & $\ldots$ & $\ldots$ & $\ldots$ & $\ldots$ & $\cdots$ & $\ldots$ \\
\hline Exponential decay & $\cdots$ & $\cdots$ & $\ldots$ & $\cdots$ & $\cdots$ & $\cdots$ \\
\hline Stellar mass independent & $\begin{array}{c}A=0.048 \\
\sigma=0.5\end{array}$ & $\begin{aligned} A & =0.07 \\
\sigma & =0.18\end{aligned}$ & $\begin{array}{c}A=0.044 \\
\sigma=0.16\end{array}$ & $\begin{array}{c}A=0.066 \\
\sigma=0.3\end{array}$ & $\begin{array}{c}A=0.098 \\
\sigma=0.26\end{array}$ & $\begin{array}{c}A=0.056 \\
\sigma=0.24\end{array}$ \\
\hline
\end{tabular}

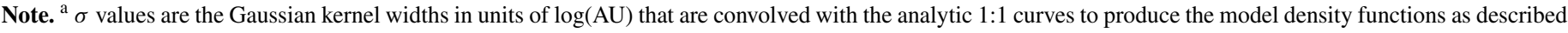

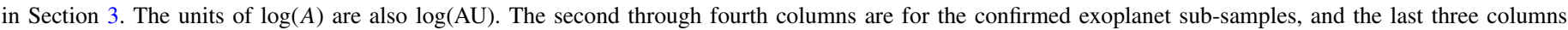
correspond to the KOIs.

Table 2

Relative Bayesian Posterior Probabilities (Unnormalized) ${ }^{\mathrm{a}}$

\begin{tabular}{|c|c|c|c|c|c|c|}
\hline Model & $M_{\mathrm{pl}}<10 M_{\oplus}$ & $10 M_{\oplus}<M_{\mathrm{pl}}<0.3 M_{J}$ & $M_{\mathrm{pl}}>0.3 M_{J}$ & $R_{\mathrm{pl}}<2 R_{\oplus}$ & $2 R_{\oplus} \leqslant R_{\mathrm{pl}} \leqslant 6 R_{\oplus}$ & $R_{\mathrm{pl}}>6 R_{\oplus}$ \\
\hline$M^{1 / 3}$ tidal model & $1.2190 \times 10^{-01}$ & $6.9976 \times 10^{07}$ & $3.8776 \times 10^{43}$ & $2.3146 \times 10^{123}$ & $4.3350 \times 10^{164}$ & $8.7087 \times 10^{28}$ \\
\hline$M^{3 / 13}$ tidal model & $7.9400 \times 10^{-02}$ & $3.6697 \times 10^{07}$ & $6.1489 \times 10^{42}$ & $6.7614 \times 10^{122}$ & $3.4503 \times 10^{164}$ & $7.7497 \times 10^{28}$ \\
\hline Magnetospheric truncation & $3.1100 \times 10^{-02}$ & $8.4200 \times 10^{06}$ & $6.4026 \times 10^{41}$ & $8.3070 \times 10^{121}$ & $8.9229 \times 10^{163}$ & $3.5485 \times 10^{28}$ \\
\hline Dust sublimation & $3.6000 \times 10^{-03}$ & $7.7860 \times 10^{-04}$ & $6.1275 \times 10^{11}$ & $1.2069 \times 10^{34}$ & $5.6110 \times 10^{35}$ & $4.7453 \times 10^{10}$ \\
\hline Power law & $2.1550 \times 10^{-01}$ & $7.2185 \times 10^{07}$ & $2.4825 \times 10^{44}$ & $3.5253 \times 10^{123}$ & $2.2953 \times 10^{164}$ & $6.3967 \times 10^{28}$ \\
\hline Uniform random & $7.5600 \times 10^{-02}$ & $3.1060 \times 10^{05}$ & $1.5013 \times 10^{19}$ & $2.8514 \times 10^{110}$ & $1.0866 \times 10^{139}$ & $2.1531 \times 10^{25}$ \\
\hline Exponential decay & $7.6600 \times 10^{-02}$ & $3.0870 \times 10^{-01}$ & $5.4692 \times 10^{12}$ & $9.1955 \times 10^{49}$ & $6.3116 \times 10^{53}$ & $1.3048 \times 10^{14}$ \\
\hline Stellar mass independent & $3.2300 \times 10^{-02}$ & $5.9364 \times 10^{06}$ & $2.5710 \times 10^{40}$ & $1.0893 \times 10^{121}$ & $9.7962 \times 10^{163}$ & $4.2242 \times 10^{28}$ \\
\hline
\end{tabular}

Note. ${ }^{\text {a }}$ The second through fourth columns are for the confirmed exoplanet sub-samples, and the last three columns correspond to the KOIs.

(2005). Each exoplanet (or candidate) in the stellar mass-log orbital semi-major axis plane is convolved with a Gaussian kernel. The width of the Gaussian kernel is set to 0.165 in stellar mass in solar units, and 0.165 in the log of the orbital semi-major axis in AU. These particular widths are chosen from the median separation between exoplanets in the confirmed exoplanet empirical distribution, which were identified to be $0.167 \log \mathrm{AU}$ in the $\log$ of the semi-major axis and $0.164 M_{\odot}$ in the stellar mass. The same kernel width is used for all empirical data sets. The kernels are summed to produce the empirical density functions shown in Figure 1. As was done for the migration halting models, each empirical distribution is evaluated numerically on a $500 \times 500$ grid evenly spaced in stellar mass from 0.1 to $1.5 M_{\odot}$ and in the $\log$ of the orbital semi-major axis in $\mathrm{AU}$ from $\log (a)=-2$ to -1 . This enables a direct subtraction of the model PDF from the empirical density function to calculate the reduced $\chi^{2}$ statistic.

Since there are no uncertainties in our empirical and theoretical distributions, to compute the reduced $\chi^{2}$ statistic we divide the square of the difference between the observations and model by the model value instead of dividing by the squared uncertainty. The model value can approximate the square of the uncertainty when the model is normalized such that the assumption of Gaussian statistics is appropriate.

\section{RESULTS}

Our results are presented in Tables 1-3. Table 1 presents the optimal parameters for a given model and empirical data set from our Bayesian analysis. Table 2 summarizes the relative posterior probabilities for a given model. Table 3 summarizes the corresponding reduced $\chi^{2}$ values. Figures 2-9 show our best-fit model density functions and residuals as a function of the model.

Excluding the power-law model for the moment, migration halting due to tidal circularization provides the best evidence and fit to the data for every data set using both the Bayesian and $\chi^{2}$ analysis, with one exception. The evidence ratio is largest for the confirmed Jovian exoplanets, which span a larger dynamic range in stellar mass relative to the KOIs. The lone exception-Neptune radii KOIs with the $\chi^{2}$ analysis-slightly favors the magnetospheric disk truncation halting mechanism. However, the difference is not statistically significant.

Comparing the 1/3rd exponent tidal halting model to the magnetospheric hole halting model, the evidence ratios are $3.9,8.3,61,28,4.9$, and 2.5 for confirmed exoplanets with $M_{\mathrm{pl}}<10 M_{\oplus}, 10 M_{\oplus}<M_{\mathrm{pl}}<0.2 M_{J}$, and $M_{\mathrm{pl}}>0.2 M_{J}$, and KOIs with $R_{\mathrm{pl}}<2 R_{\oplus}, 2 R_{\oplus}<R_{\mathrm{pl}}<6 R_{\oplus}$, and $R_{\mathrm{pl}}>$ $6 R_{\oplus}$, respectively. Thus, our Bayesian analysis disfavors the models of halting interior to the magnetospheric dust truncation radius, halting interior to the dust sublimation radius, halting at a constant radius independent of stellar mass, and a uniform random distribution in both the semi-major axis and the log of the semi-major axis.

The power-law model has the largest posterior probabilities for the confirmed exoplanet sub-samples, and is within a factor of two of the most favored models (tidal circularization) for the KOI sub-samples. The power-law model is clearly favored over the stellar-mass-independent model, with posterior probability 

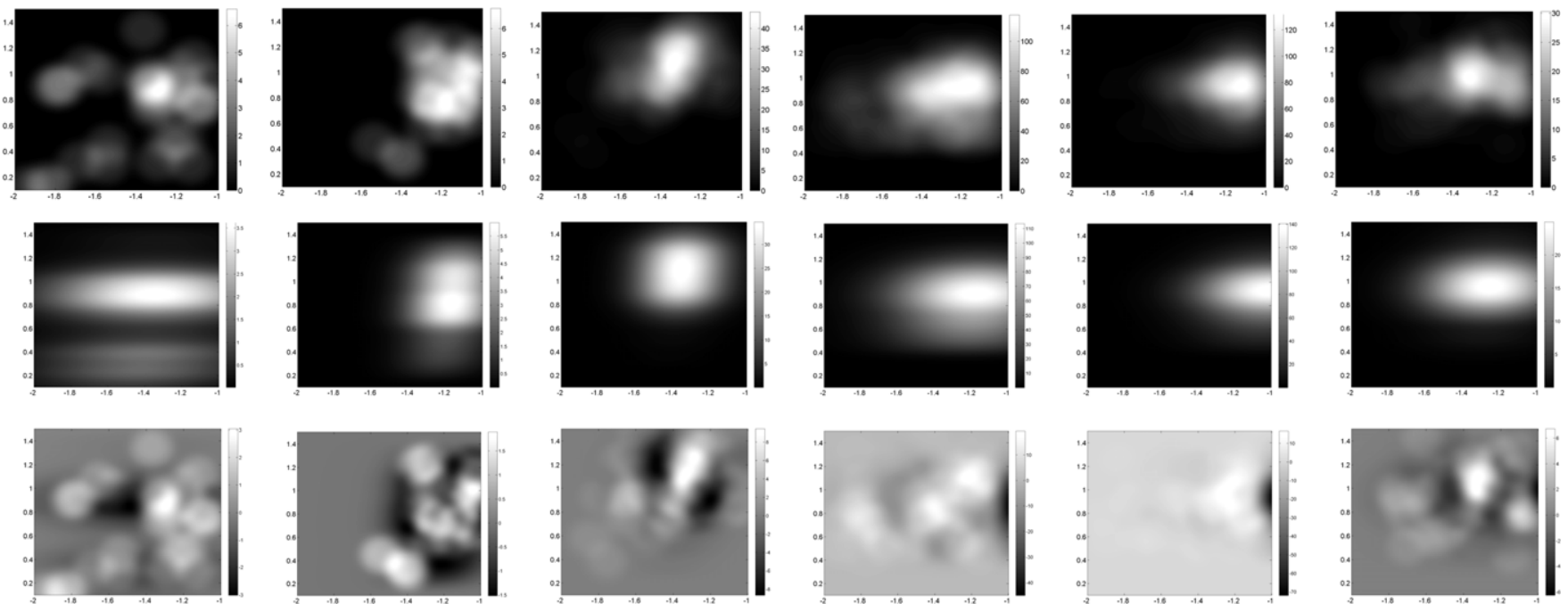

Figure 2. Top: empirical density functions as in the second row of Figure 1. Middle: model probability density function with optimal parameters in Table 1 for the halting model at the interior 1:2 orbital resonance with the magnetospheric disk truncation radius. Bottom: subtracted residuals.
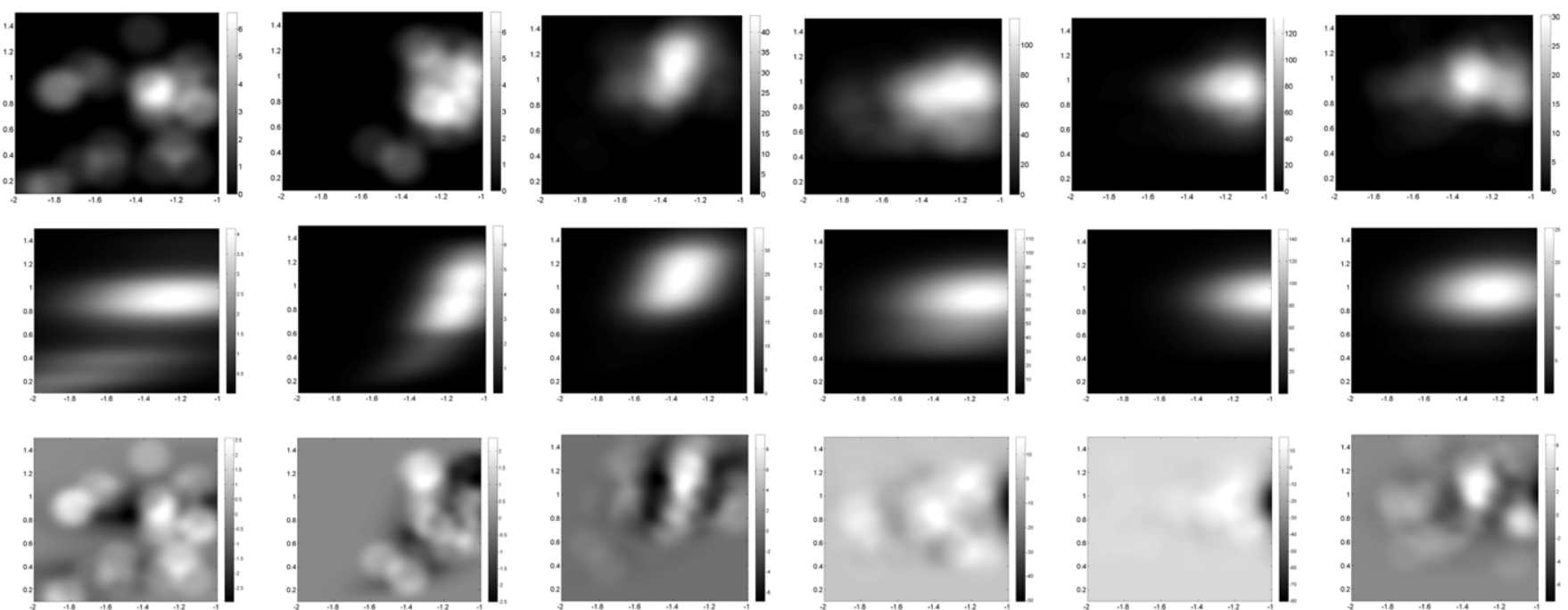

Figure 3. Same as Figure 2 for the power-law halting model, with an optimized exponent free parameter.

Table 3

Reduced $\chi^{2}$ Values ${ }^{\mathrm{a}}$

\begin{tabular}{|c|c|c|c|c|c|c|}
\hline Model & $M_{\mathrm{pl}}<10 M_{\oplus}$ & $10 M_{\oplus}<M_{\mathrm{pl}}<0.3 M_{J}$ & $M_{\mathrm{pl}}>0.3 M_{J}$ & $R_{\mathrm{pl}}<2 R_{\oplus}$ & $2 R_{\oplus} \leqslant R_{\mathrm{pl}} \leqslant 6 R_{\oplus}$ & $R_{p l}>6 R_{\oplus}$ \\
\hline$M^{1 / 3}$ tidal model & 0.51739 & 0.32685 & 0.89054 & 1.0987 & 1.2792 & 0.35351 \\
\hline$M^{3 / 13}$ tidal model & 0.54308 & 0.41063 & 1.6656 & 1.1376 & 1.2416 & 0.35557 \\
\hline Magnetospheric truncation & 0.57783 & 0.39862 & 2.4932 & 1.2987 & 1.2235 & 0.37129 \\
\hline Dust sublimation & 0.78871 & 1.8692 & 5.4704 & 13.1747 & 18.9103 & 2.7711 \\
\hline Power law & 0.5709 & 0.39799 & 0.55665 & 1.1233 & 1.3821 & 0.37203 \\
\hline Uniform random & 0.88506 & 0.7415 & 4.7696 & 3.7036 & 3.8442 & 1.1901 \\
\hline Exponential decay & 0.72727 & 1.6272 & 5.5312 & 11.078 & 15.8296 & 2.4884 \\
\hline Stellar mass independent & 0.62176 & 0.51691 & 5.3648 & 1.518 & 1.4775 & 0.42532 \\
\hline
\end{tabular}

Note. ${ }^{\text {a }}$ The second through fourth columns are for the confirmed exoplanet sub-samples, and the last three columns correspond to the KOIs.

ratios ranging from 1.5 to $10^{4}$. This result is weakest for the Jovian KOIs, but strongest for the confirmed Jovian exoplanets, and we again attribute that to the lack of dynamic range in stellar mass for the KOIs but also may be partially attributable to a high false-positive rate for Jovian KOIs.

For both the confirmed exoplanets and KOIs, smaller exoplanets favor both steeper power laws and larger dispersion (Gaussian Kernel width) than Jovian planets, and in all cases the exponent is larger than either the magnetospheric disk truncation and exoplanet tidal excitation model exponents. This result is intriguing, and we speculate that there may be additional physics in the tidal theories to halt planet migration as a function of exoplanet mass/density.

Howard et al. (2012) report the decreasing planet frequency toward smaller orbital separations within $\sim 0.04$ AU. Our analysis confirms the observed dearth of Kepler exoplanet 

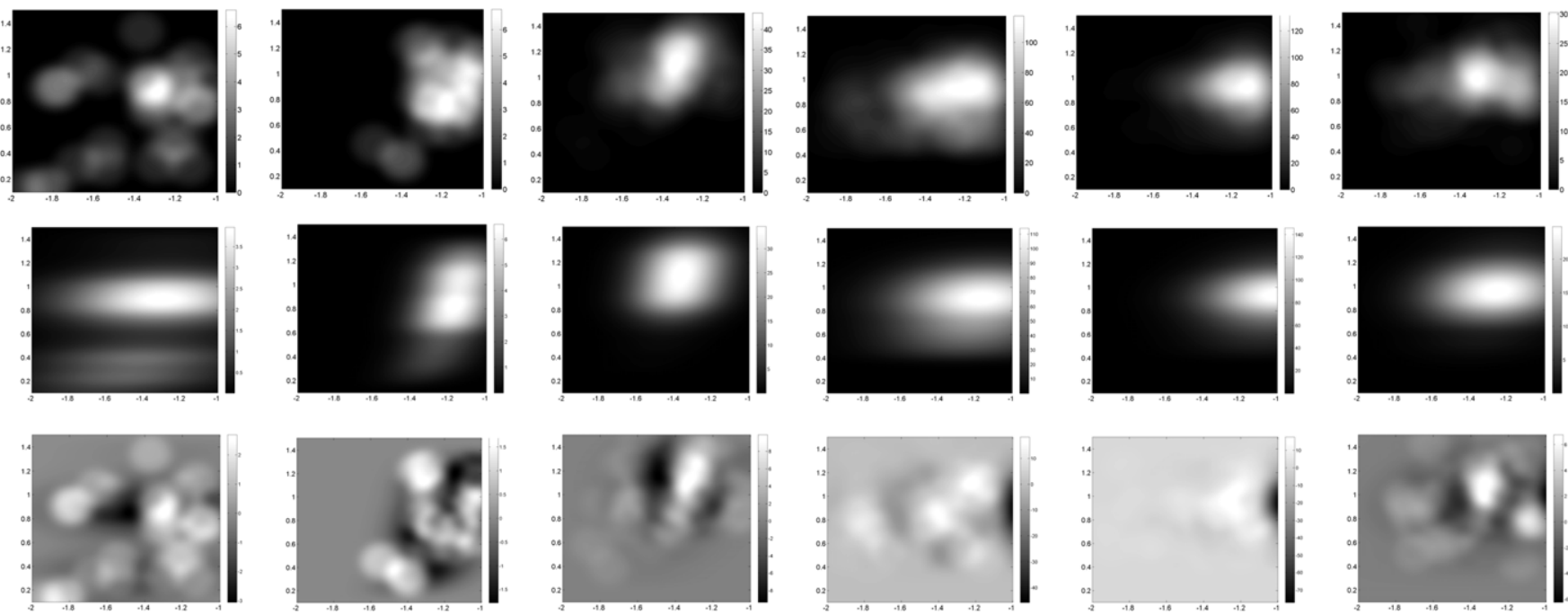

Figure 4. Same as Figure 2 for the Roche radius a $\propto M^{1 / 3}$ tidal halting model.
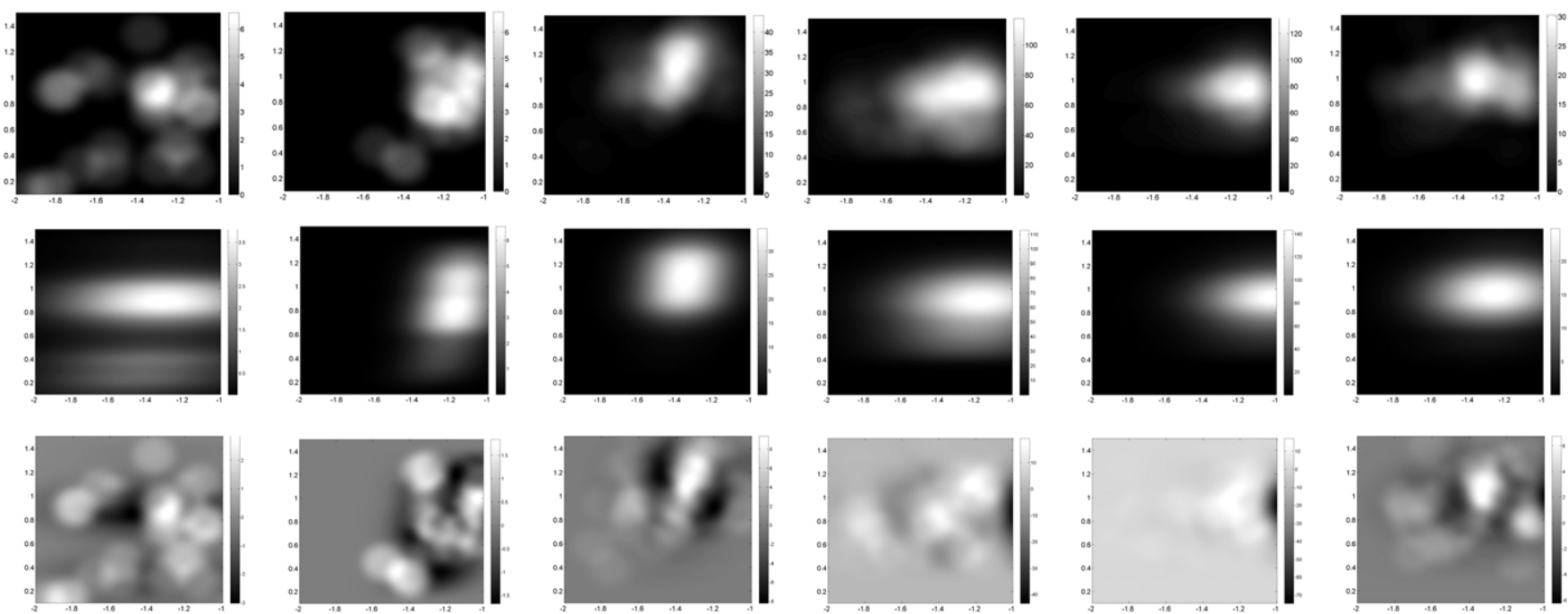

Figure 5. Same as Figure 2 for the $a \propto M^{3 / 13}$ tidal halting model.
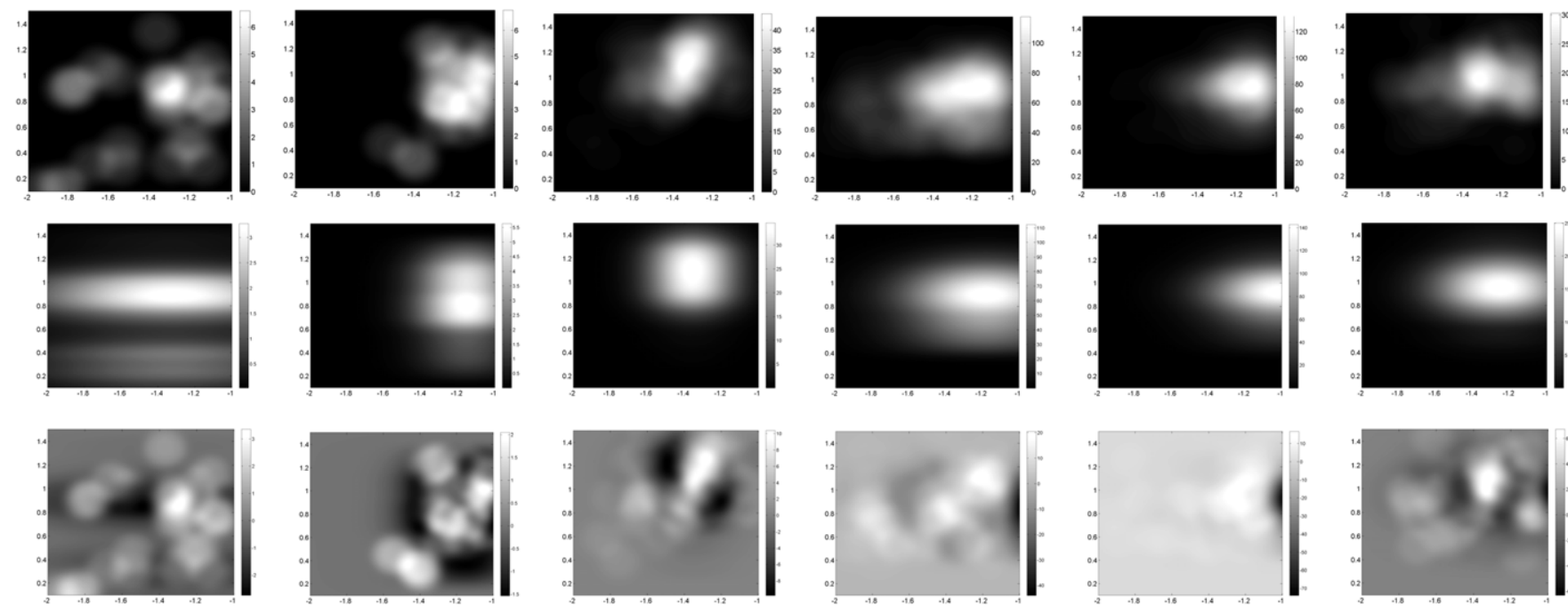

Figure 6. Same as Figure 2 for the stellar-mass-independent halting model at a constant semi-major axis. 

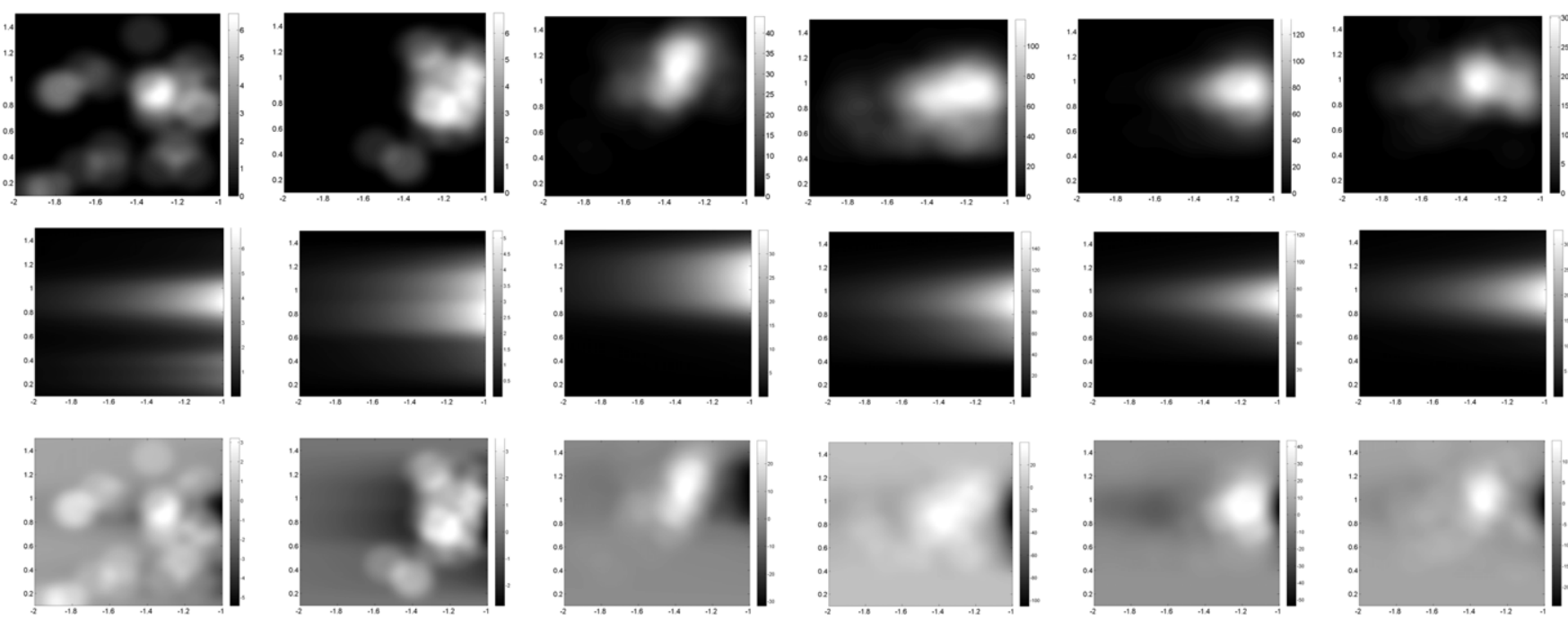

Figure 7. Same as Figure 2 for the uniform random halting model.
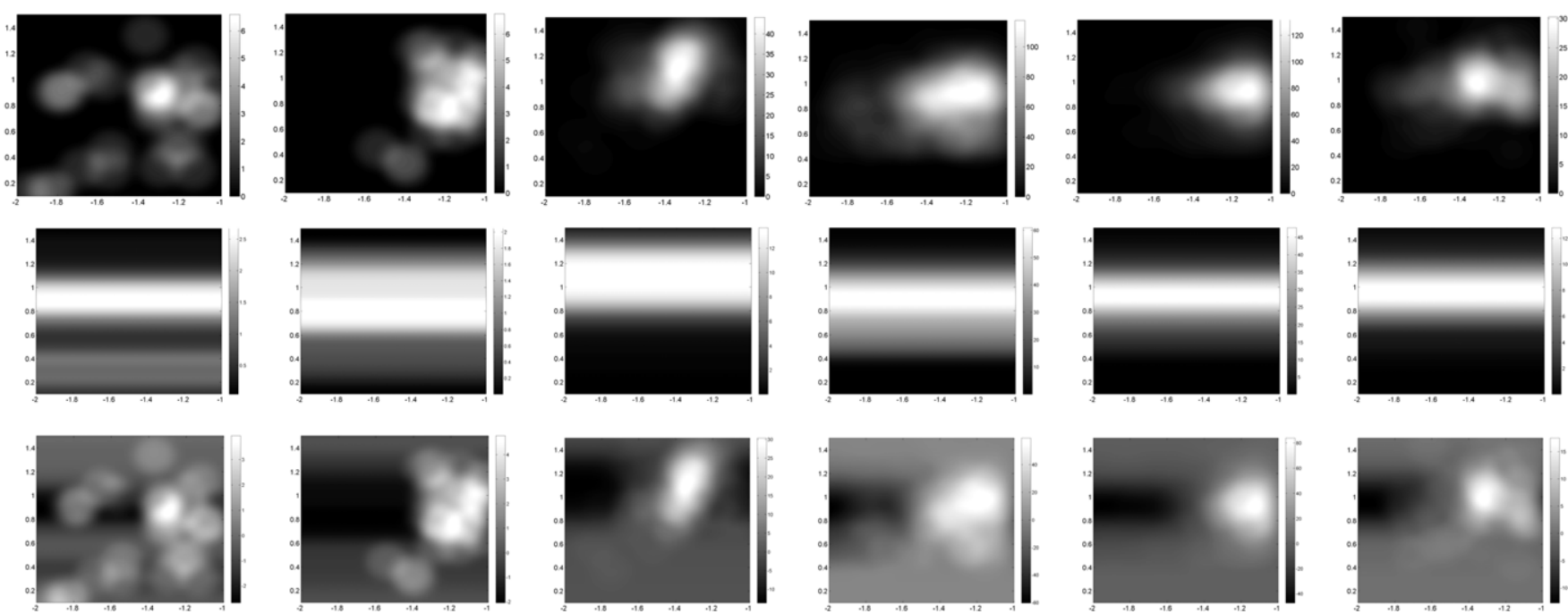

Figure 8. Same as Figure 2 for the exponential decay with semi-major axis tidal halting model.
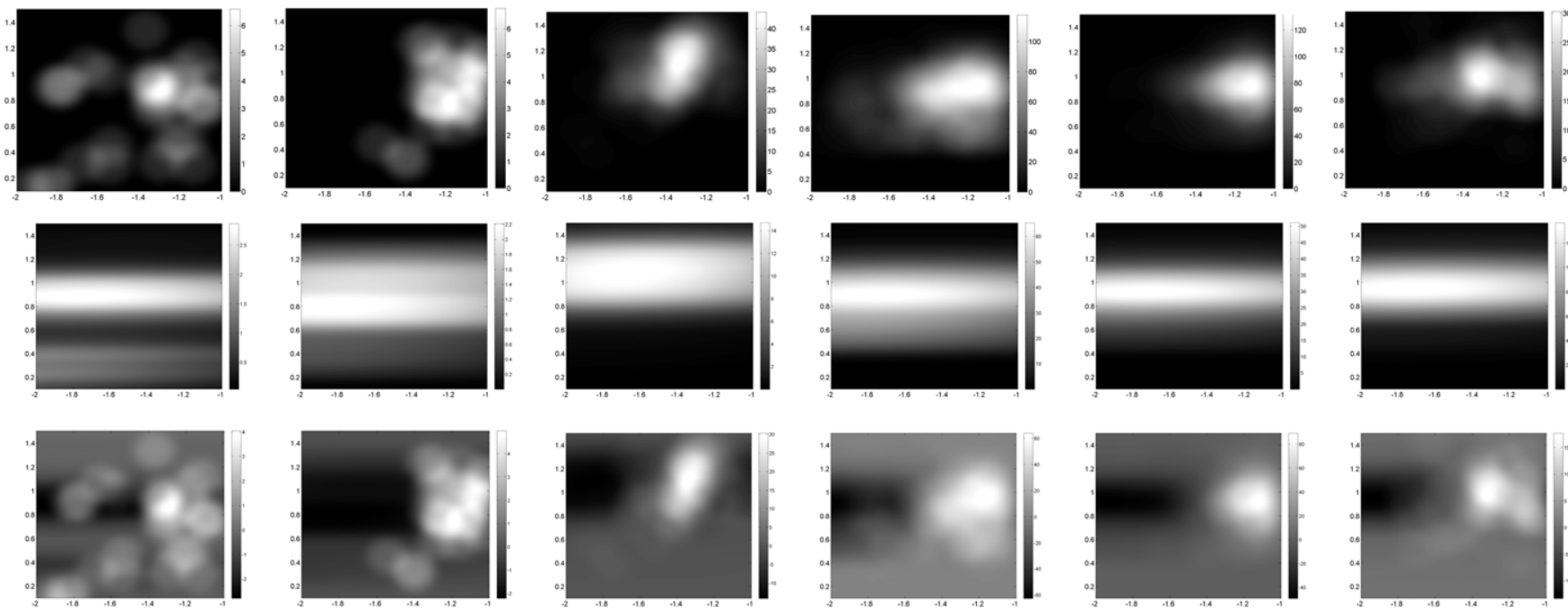

Figure 9. Same as Figure 2 for the halting model at the interior 1:2 orbital resonance with the dust sublimation radius. 
candidates at these small orbital radii by rejecting the uniform random and exponential decay halting models.

The subtle differences between the best models are not clear "by eye" in Figures 2-5. However, the $\chi^{2}$ test demonstrates a slight preference for the tidal circularization halting models for all data sets save the Neptune-like KOIs. Our Bayesian analysis does not rely on a Gaussian kernel estimate of the empirical density function used in the $\chi^{2}$ analysis (Section 4.2), and this may partially account for the weaker statistical significance between the two approaches.

The best-fit magnetospheric disk truncation model magnetic field strengths range from $1-4.4 \mathrm{kG}$ for each of our data sets, consistent with observed $\mathrm{T}$ Tauri magnetic field strengths given our assumptions about the mass accretion rates in Section 3.2 (Johns-Krull et al. 2003; Eisner et al. 2005). However, this is likely coincidental. For the model of halting at a constant semimajor axis independent of the stellar mass, the preferred mean value of $a$ ranges from 0.044 to $0.098 \mathrm{AU}$ as expected for the close-in exoplanets.

Finally, we find that the best-fit values for the Gaussian kernel width $\sigma$ in the $\log$ of the semi-major axis provide a reasonable prescription for the data as evidenced by the reduced $\chi^{2}$ values. We do not attempt to explain the additional non-Gaussian substructure in the estimated empirical PDF that can be seen in Figure 1 for all data sets and in the model residuals in the subsequence figures, but note that such sub-structure could point to multiple migration halting mechanisms operating.

\section{CONCLUSIONS}

We use the empirical distribution of confirmed exoplanets and Kepler planet candidates in the host stellar mass-exoplanet orbital semi-major axis plane as a diagnostic for migration halting mechanisms. Migration halting from tidal circularization provides the best posterior probabilities for all empirical samples, favored by factors of 2.5-61 for the different sub-samples investigated herein when compared to halting at the 1:2 interior resonance with the magnetospheric disk truncation radius. We can rule out migration halting at the 1:2 interior resonance with the dust sublimation radius, a uniform random halting radius, and an exponential decay halting radius as viable models for the majority of observed close-in exoplanets. Our generalized power-law model favors a dependence of the halting distance with stellar mass that is stronger than predicted from tidal dissipation theories, and clearly rules out the independence of the halting distance on stellar mass, with posterior probability ratios ranging from 1.5 to $10^{4}$. The favoring of a stronger power-law dependence than predicted for the tidal halting model suggests that future theoretical work may be needed to better reproduce the observed sub-structure in the empirical distribution of exoplanets as a function of semi-major axis and stellar mass.

The authors thank the referee Dan Fabrycky for his patience, corrections, and comments to our analysis, which substantially improved this work. This research has made use of the NASA Exoplanet Archive, which is operated by the California Institute of Technology, under contract with the National Aeronautics and Space Administration under the Exoplanet Exploration Program. This research has made use of the Exoplanet Orbit Database and the Exoplanet Data Explorer at exoplanets.org. The authors also thank Thayne Currie for his useful (and rapid turnaround) comments on the manuscript. We also acknowledge the utility of the Bayesian statistics class taught by John Johnson at Caltech.

\section{REFERENCES}

Akeson, R., Chen, X., Ciardi, D., et al. 2013, PASP, submitted Albrecht, S., Winn, J. N., Carter, J. A., Snellen, I. A. G., \& de Mooij, E. J. W. 2011, ApJ, 726, 68

Albrecht, S., Winn, J. N., Johnson, J. A., et al. 2012, ApJ, 757, 18

Arras, P., Burkart, J., Quataert, E., \& Weinberg, N. 2012, MNRAS, 422, 1761

Baraffe, I., Chabrier, G., Allard, F., \& Hauschildt, P. 1998, A\&A, 337, 403

Batalha, N., Borucki, W. J., Koch, D. G., et al. 2010a, ApJL, 713, L109

Batalha, N., Rowe, J. F., Bryson, S. T., et al. 2013, ApJS, 204, 24

Batalha, N., Rowe, J. F., Gilliland, R. L., et al. 2010b, ApJL, 713, L103

Borucki, W., Koch, D., Basri, G., et al. 2010, Sci, 327, 977

Borucki, W., Koch, D. G., Basri, G., et al. 2011, ApJ, 736, 19

Brown, T., Latham, D. W., Everett, M. E., \& Esquerdo, G. A. 2011, AJ, 142,112

Chiang, E. I., \& Goldreich, P. 1997, ApJ, 490, 368

Currie, T. C., Lada, C. J., Plavchan, P., et al. 2009, ApJ, 698, 1

D’Alessio, P., Calvet, N., Hartmann, L., Franco-Hernández, R., \& Servín, H. 2006, ApJ, 638, 314

Dawson, R., \& Murray-Clay, R. A. 2013, ApJL, in press (arXiv:1302.6244)

Dawson, R., Murray-Clay, R. A., \& Johnson, J. A. 2013, ApJ, submitted (arXiv:1211.0554)

Eisner, J., Hillenbrand, L. A., White, R. J., Akeson, R. L., \& Sargent, A. I. 2005, ApJ, 623, 952

Endl, M., Cochran, W. D., Krster, M., et al. 2006, ApJ, 649, 436

Ford, E., \& Rasio, F. 2006, ApJL, 638, L45

Greene, T., \& Lada, C. 1996, AJ, 112, 2184

Guillochon, J., Ramirez-Ruiz, E., \& Lin, D. N. C. 2011, ApJ, 732, 74

Hale, A. 1994, AJ, 107, 306

Howard, A., Marcy, G. W., Bryson, S. T., et al. 2012, ApJS, 201, 15

Johns-Krull, C. M., Valenti, J. A., \& Gaord, A. D. 2003, RMxAA, 18, 38

Johnson, J. A., Clanton, C., Howard, A. W., et al. 2011, ApJS, 197, 26

Jura, M., Malkan, M., White, R., et al. 1998, ApJ, 505, 897

Kaib, N., Raymond, S., \& Duncan, M. 2011, ApJL, 742, L24

Konigl, A. 1991, ApJL, 370, L39

Kozai, Y. 1962, AJ, 67, 591

Kuchner, M. J., \& Lecar, M. 2002, ApJL, 574, L87

Lai, D. 2012, MNRAS, 423, 486

Lin, D. N. C., Bodenheimer, P., \& Richardson, D. C. 1996, Natur, 380, 606

Lubow, S. H., \& Ida, S. 2010, in Exoplanets, ed. S. Seager (Tucson, AZ: Univ. Arizona Press), 347

Marcy, G., Butler, R. P., Williams, E., et al. 1997, ApJ, 481, 926

Marcy, G., \& Butler, P. 1998, ARA\&A, 36, 57

Marshall, P., Rajguru, N., \& Slosar, A. 2006, PhRvD, 73, 7302

Matsumura, S., Peale, S., \& Rasio, F. 2010, ApJ, 725, 1995

Menou, K., \& Goodman, J. 2004, ApJ, 606, 520

Meyer, M., Calvet, N., \& Hillenbrand, L. 1997, AJ, 114, 288

Morton, T., \& Johnson, J. 2011, ApJ, 729, 138

Muzerolle, J., Hillenbrand, L., Calvet, N., Briceño, C., \& Hartmann, L. 2003, ApJ, 592, 266

Nagasawa, M., \& Ida, S. 2011, ApJ, 742, 72

Naoz, S., Farr, W. M., Lithwick, Y., Rasio, F. A., \& Teyssandier, J. 2011, Natur, 473, 187

Narita, N., Hirano, T., Sanchis-Ojeda, R., et al. 2010, PASJ, 62, L61

Plavchan, P., Bilinski, C. G., \& Currie, T. C. 2013, PASP, submitted (arXiv:1203.1887)

Plavchan, P., Jura, M., Kirkpatrick, J. D., Curti, R. M., \& Gallagher, S. C. 2008, ApJS, 175, 191

Prato, L., \& Weinberger, A. J. 2007, in Planets in Binary Star Systems, ed. N. Haghighipour (Berlin: Springer), 1

Raymond, S., Mandell, A., \& Sigurdsson, S. 2006, Sci, 313, 1413

Robitaille, T. P., Whitney, B. A., Indebetouw, R., \& Wood, K. 2007, ApJS, 169,328

Robitaille, T. P., Whitney, B. A., Indebetouw, R., Wood, K., \& Denzmore, P. 2006, ApJS, 167, 256

Santerne, A., Díaz, R. F., Moutou, C., et al. 2012, A\&A, 545A, 76

Siess, L., Dufour, E., \& Forestini, M. 2000, A\&A, 358, 593

Silverstone, M., Meyer, M. R., Mamajek, E. E., et al. 2006, ApJ, 639, 1138

Tanaka, H., Takeuchi, T., \& Ward, W. 2002, ApJ, 565, 1257

Terquem, C. 2003, MNRAS, 341, 1157

Thies, I., Kroupa, P., Goodwin, S. P., Stamatellos, D., \& Whitworth, A. P. 2011, MNRAS, 417, 1817

Triaud, A. H. M. J., Collier Cameron, A., Queloz, D., et al. 2010, A\&A, 524,25

Tsang, D. 2011, ApJ, 741, 109

Ward, W. 1997a, ApJL, 482, L211 
Ward, W. 1997b, Icar, 126, 261

Wasserman, L. 2005, All of Non-Parameteric Statistics (Berlin: Springer)

Watson, C. A., Littlefair, S. P., Diamond, C., et al. 2011, MNRAS, 413, L71

Winn, J., Fabrycky, D., Albrecht, S., \& Johnson, J. A. 2010, ApJL, 718, L145
Wright, J. T., Fakhouri, O., Marcy, G. W., et al. 2011, PASP, 123, 412

Wu, Y., \& Lithwick, Y. 2011, ApJ, 735, 109

Wu, Y., \& Murray, N. 2003, ApJ, 589, 605

Wu, Y., Murray, N., \& Ramsahai, J. 2007, ApJ, 670, 820 